\title{
EL POLEN DE ESPECIES DEL GÉNERO NICOTIANA (SOLANACEAE) PRESENTES EN CHILE: EVALUACIÓN DE LA UTILIDAD DE SUS CARACTERES MORFOLÓGICOS COMO BIOMARCADORES EN ESTUDIOS ARQUEOLÓGICOS
}

\author{
KATHY COLLAO-ALVARADO ${ }^{1}$, MARÍA TERESA PLANELLA² y HERMANN M. NIEMEYER ${ }^{1}$
}

\begin{abstract}
Summary: Pollen of species from the genus Nicotiana (Solanaceae) present in Chile: Evaluation of the usefulness of morphological characters as biomarkers in archaeological studies. The alkaloid nicotine is taken up when Nicotiana plants are smoked, chewed or snuffed. The use of tobacco has been detected by the presence of nicotine in the residues of archaeological pipes and also in mummified human hair; however, the lack of species-specific markers has made it impossible to determine the Nicotiana species involved. Pollen has been used as a reliable biomarker for identifications at the family or genus levels. We report herein the study of pollen from 10 native species of Nicotiana from Chile, including the wild $N$. glauca and the cultivated $N$. tabacum, using both optical and scanning electron microscopy. The results show minimal interspecific qualitative differences. Although the mean values of ratio polar and equatorial diameters in an equatorial view, colpus length and exine thickness show statistically significant differences, the data variance does not allow its use in the identification of Nicotiana at the species level and therefore the usefulness of pollen as a biomarker is limited at the species level.
\end{abstract}

Key words: Tobacco, nicotin, biomarker, pollen.

\begin{abstract}
Resumen: Diversas sociedades americanas prehispánicas han utilizado el tabaco (Nicotiana spp.) en contextos sagrados, medicinales o recreativos. Las especies de Nicotiana contienen nicotina, alcaloide que es ingerido al fumar, masticar o inhalar la planta. El uso del tabaco se ha detectado a través de la presencia de nicotina en residuos de pipas de origen arqueológico y en cabello de individuos momificados; sin embargo, la carencia de marcadores especie-específicos ha imposibilitado conocer la especie de Nicotiana de procedencia. El polen ha sido utilizado como biomarcador confiable para identificaciones al nivel de familia y género. Se presenta un estudio mediante microscopía óptica y electrónica de barrido del polen de 10 especies de Nicotiana nativas de Chile, la especie alóctona asilvestrada N. glauca y la especie cultivada $N$. tabacum. Se encontraron mínimas diferencias interespecíficas en parámetros cualitativos. Aunque los promedios de los valores de ciertos parámetros (cociente entre los diámetros polar y ecuatorial, ambos en vista ecuatorial, la longitud de los colpos largos y el espesor de la exina) muestran diferencias estadísticamente significativas, la varianza de los datos no permite su uso en la identificación al nivel de especie. En consecuencia, la utilidad del polen como biomarcador al nivel de especie es limitada.
\end{abstract}

Palabras clave: Tabaco, nicotina, biomarcador, polen.

\section{INTRODUCCIÓN}

El consumo de sustancias psicoactivas de origen vegetal, particularmente de tabaco (Nicotiana spp., Solanaceae), es una costumbre arraigada

\footnotetext{
1 Facultad de Ciencias, Universidad de Chile, Las Palmeras 3425, 7800024 Santiago, Chile; k.collao.a@ gmail.com (autor corresponsal).

2 Sociedad Chilena de Arqueología, El Amancai 505, 7591424 Santiago, Chile.
}

en numerosas poblaciones prehispánicas de toda América (Wilbert 1987, Torres 1996). Estudios etnográficos han demostrado que en numerosos pueblos originarios americanos, shamanes y sanadores consumen tabaco en contextos rituales y medicinales aunque también los individuos pueden hacerlo por placer (Latcham 1924, Pérez \& Gordillo 1993, Winter 2000).

El uso del tabaco ha sido determinado a través de la presencia de nicotina o de microrrestos botánicos de Nicotiana en residuos de pipas, micromorteros y 
pistilos provenientes de diversos sitios del área Surandina (Capparelli et al. 2006, Andreoni et al. 2012, Quiroz et al.2012, Planella et al. 2012a, Echeverría et al. 2014, Planella et al. 2014), en residuos de pipas de sitios en Norteamérica (Rafferty 2002, 2006, Eerkens et al. 2012, Tushingham et al. 2013), y en el cabello de individuos prehispánicos momificados en Sudamérica (Musshoff et al. 2009, Echeverría \& Niemeyer 2013) .

La búsqueda de marcadores de identidad al nivel de especie ha sido abordada en Nicotiana utilizando distintos enfoques y técnicas. Entre ellos, los marcadores arqueobotánicos, particularmente microfósiles como silicofitolitos y granos de almidón han sido usados para demostrar presencia de Nicotiana en residuos de pipas arqueológicas (Capparelli et al. 2006, Andreoni et al. 2012; Quiroz et al. 2012); sin embargo, la morfología de los granos de almidón no es enteramente confiable para la identificación a nivel de especie, a diferencia de lo que ocurre con las identificaciones basadas en la morfología de los tricomas (Quiroz 2014; Belmar et al. 2015). Por otro lado, recientemente, fue demostrado que las semillas de Nicotiana halladas en sitios arqueológicos conservan características morfológicas que pueden ser utilizadas para su identificación taxonómica al nivel de especie (Planella et al. 2012b).

Una posibilidad aún no explorada es el uso del polen de Nicotiana como biomarcador. Particularmente, el polen de tabaco podría encontrarse en diversos contextos, debido a que la superficie de las hojas de Nicotiana sustenta abundantes tricomas (Goodspeed 1954), que actúan como trampas naturales del polen ambiental y del suyo propio. Por ejemplo, Donaldson \& Stephens (2010) recuperaron granos de polen desde hojas de tabaco utilizadas en la manufactura de cigarrillos; una vez identificados al nivel de familia botánica, el origen geográfico del tabaco pudo ser determinado sobre la base de la identificación de las especies que acompañaban a Nicotiana. Más relevante aún para la identificación del uso de Nicotiana en material arqueológico, Bryant et al. (2012) obtuvieron granos de polen desde residuos carbonizados de pipas.

En consecuencia, los objetivos de este trabajo fueron: i) realizar una caracterización morfológica del polen utilizando microscopía óptica y electrónica de barrido de las especies de Nicotiana presentes en
Chile (Marticorena \& Quezada 1985): Nicotiana acuminata, $N$. cordifolia, $N$. corymbosa, N. linearis, $N$. longibracteata, $N$. miersii var. miersii, $N$. pauciflora, $N$. petunioides, $N$. solanifolia y $N$. undulata, la especie alóctona asilvestrada N. glauca y la especie cultivada $N$. tabacum, y ii) determinar los cambios morfológicos del polen producidos como consecuencia de la calcinación con el fin de efectuar comparaciones con el polen calcinado presente en los restos arqueológicos como es en el caso de las pipas para fumar.

\section{Materiales y Métodos}

Se confeccionó una colección de referencia con polen de 12 especies de Nicotiana presentes en Chile (Marticorena \& Quezada 1985), obtenido a partir de flores y anteras provenientes de material depositado en el Herbario de la Universidad de Concepción, Chile (CONC). De cada especie se analizaron 25 granos de polen.

La muestra de polen fue acetolizada según Erdtman (1952). Los granos de polen fueron montados y sellados con gelatina glicerinada. Las muestras fueron rotuladas y almacenadas en el Laboratorio de Química Ecológica de la Universidad de Chile.

Los granos fueron fotografiados en vistas polar y ecuatorial con una cámara digital (MSHOT MD90) incorporada a un microscopio óptico (Olympus YZ). Para un estudio más detallado de la ornamentación de los granos de polen acetolizados se utilizó microscopía electrónica de barrido (Carl Zeiss EVO MA10) luego de metalización con oro (Cresington 108 auto) bajo un vacío de $c a$. 0,05 mbar por 70 segundos y una corriente de $20 \mu \mathrm{A}$.

Las mediciones de los granos de polen observados bajo MO fueron realizadas con los programas Axiovision y MSHOT Digital Imaging System y las mediciones bajo MEB con el programa Smart SEM. La terminología utilizada sigue a Punt et al. (2007). Las descripciones de los granos bajo MO comprenden: ámbito (vista polar), aberturas (tipo, número, posición, longitud de colpos largos, diámetro ecuatorial $(\mathrm{E})$, diámetro polar $(\mathrm{P})$, espesor y ornamentación de la exina, y forma determinada a partir de la relación P/E. El MEB se utilizó para describir detalles de la ornamentación.

Para evaluar el efecto de la calcinación, siete 


\section{K. Collao-Alvarado et al. - Polen de especies de Nicotiana en Chile}

muestras de polen de $N$. tabacum de $10 \mathrm{mg}$ cada fueron sometidas a diferentes temperaturas $\left(150^{\circ}, 165^{\circ}, 185^{\circ}, 205^{\circ}, 230^{\circ}, 290^{\circ}\right.$ y $\left.320^{\circ} \mathrm{C}\right)$ por 30 minutos; luego los granos de polen fueron fotografiados con MEB.

Las doce especies de tabaco utilizadas como material de referencia se distribuyen en Chile y corresponden a: Nicotiana acuminata (Graham) Hook., Región de Valparaíso, Prov. Aconcagua, Los Andes, 22-III-1989, Dr. K. Behn 22087 (CONC); Nicotiana cordifolia Phil., Región de Valparaíso, Archipiélago de Juan Fernández, Isla Más Afuera, Quebrada Las Casas, 19-I-1941, Leg. P. Aravena 48991 CONC); Nicotiana corymbosa Remy, Región de Coquimbo, Prov. Elqui, Canchas de esquí, 22-II-1988, Leg. F. Squeo 22065 (CONC); Nicotiana glauca Graham, Región de Coquimbo, Chorrillos, a orillas de estero, 25-II-1941, Leg. C. Junge s.n. (CONC); Nicotiana linearis Phil., Región de Coquimbo, Ovalle, El Reloj, 5-IX1949, leg. C. Jiles 31516 (CONC); Nicotiana longibracteata Phil., Región de Antofagasta, Prov. El Loa, entre Laguna Tuayatu y Socaire, 5-V-1999, R. Rodríguez y E. Ruiz 3604 (CONC); Nicotiana miersii var. miersii Remy, Región de Coquimbo, Depto. Ovalle, Seron, 30-VIII-1957, Leg. C. Jiles 31530 (CONC); Nicotiana pauciflora Remy, Región de Coquimbo, Prov. Elqui, Cordillera de Paihuano, orilla de río, XII-1942, E. Gajardo 22082 (CONC); Nicotiana petunioides (Griseb) Milán, Región de Antofagasta, subida desde Río Frío a Aguas Calientes entre Cuesta de Lata y el salar, 18-I-1994, Leg. M. Arroyo, R. Leonard y J. Cuevas 122113 (CONC); Nicotiana solanifolia Walp., Región de Antofagasta, Quebrada Sepultura, 3-X1991, leg. M. Quezada y E. Ruiz 121233 (CONC); Nicotiana tabacum L. cv. BY 64, ProfiGen do Brasil Ltda. Sta. Cruz do Sul, RS, Brasil; Nicotiana undulata Ruiz et Pav., Región de Tarapacá, Prov. Parinacota, Quebrada Aroma, X-1987, Leg. G. González 126966 (CONC).

\section{Resultados}

Los granos de polen de las especies de tabaco son mónades, isopolares, prolato-esferoidales, subprolatos o prolatos, ámbito circular y en algunos casos semiangular, de tamaño grande, tricolporados y con presencia de colpos largos que miden en promedio $62,4 \mu \mathrm{m}$ de longitud llegando casi a ambos polos. La exina es tectada y de espesor variable.

Con MEB se confirmó que en algunas especies la ornamentación se transforma en psilada hacia el apocolpium y que sólo a elevados aumentos (45.000x) es posible distinguir granulaciones al interior de los colpos en vista polar.

Descripción palinológica de las especies de tabaco

Nicotiana acuminata (Graham) Hook. (Fig. 1.A-C).

MO: Granos subprolatos $(\mathrm{P}=67-84 \mu \mathrm{m}, \mathrm{E}=56-74 \mu \mathrm{m}, \mathrm{P} /$ $\mathrm{E}=0,96-1,37 \mu \mathrm{m})$, ámbito circular. Colpos largos varían entre 59 y $76 \mu \mathrm{m}$ de longitud. Exina tectada, de 1,6-2,6 $\mu \mathrm{m}$ de espesor. Ornamentación estriada-escábrida.

MEB: Ornamentación estriada (Fig. 5.C), presencia de gránulos al interior de los colpos (Fig. 5.D) y apocolpium psilado (Fig. 5.E).

Distribución geográfica en Chile: Regiones II, III, IV, V, VI, VII, VIII, Región Metropolitana de Santiago.

\section{N. cordifolia Phil. (Fig. 1.D-F)}

MO: Granos subprolatos $(\mathrm{P}=59-77 \mu \mathrm{m}, \mathrm{E}=46-60 \mu \mathrm{m}, \mathrm{P} /$ $\mathrm{E}=1,02-1,63 \mu \mathrm{m})$, ámbito circular o semi angular. Colpos largos varían entre 48 y $76 \mu \mathrm{m}$ de longitud. Exina tectada, de 1,6-2,8 $\mu \mathrm{m}$ de espesor. Ornamentación estriadareticulada heterobrocada.

MEB: Ornamentación rugulada; con magnificación 67.000x se observan perforaciones en la pared (Fig. 5.G)

Distribución geográfica en Chile: Región V.

N. corymbosa Remy (Fig. 1. G-I)

MO: Granos subprolatos $(\mathrm{P}=65-84 \mu \mathrm{m}, \mathrm{E}=46-71 \mu \mathrm{m}, \mathrm{P} /$ $\mathrm{E}=1,05-1,80 \mu \mathrm{m})$, ámbito circular.

Colpos largos varían entre 52 y $76 \mu \mathrm{m}$ de longitud. Exina tectada de 1,6-2,8 $\mu \mathrm{m}$ de espesor. Ornamentación estriada.

MEB: Ornamentación rugulada-estriada, psilada hacia el apocolpium (Fig. 5.H); con magnificación 20.000x se observa verrucoide (Fig. 5.I)

Distribución geográfica en Chile: II, III, IV, V, VII, Región Metropolitana de Santiago.

N. glauca Graham (Fig. 2.A-C)

MO: Granos prolatos $(\mathrm{P}=54-83 \mu \mathrm{m}, \mathrm{E}=39-54 \mu \mathrm{m}, \mathrm{P} / \mathrm{E}=$ 1,07-1,8 $\mu \mathrm{m})$, ámbito circular.

Colpos largos varían entre 44 y $71 \mu \mathrm{m}$ de longitud. Exina tectada de 1,9-2,9 $\mu \mathrm{m}$ de espesor. Ornamentación 
estriada-escábrida.

MEB: Ornamentación estriada-rugulada (Fig. 6.B).

Distribución geográfica: Regiones I, II, III, IV, V, Región Metropolitana de Santiago.

\section{N. linearis Phil (Fig. 2. D-F)}

MO: Granos subprolatos $(\mathrm{P}=57-84 \mu \mathrm{m}, \mathrm{E}=47-81 \mu \mathrm{m}, \mathrm{P} /$ $\mathrm{E}=0,9-1,46 \mu \mathrm{m})$, ámbito circular o semiangular. Colpos largos varían entre 55 y $76 \mu \mathrm{m}$ de longitud. Exina tectada de 1,9-3,6 $\mu \mathrm{m}$ de espesor. Ornamentación estriada y reticulada-heterobrocada.

MEB: Ornamentación rugulada; con magnificación 43.830x se observa verrucoide (Fig. 6.D).

Distribución geográfica en Chile: Regiones IV, VII

\section{N. longibracteata Phil (Fig. 2.G-I)}

MO: Granos subprolatos $(\mathrm{P}=57-81 \mu \mathrm{m}, \mathrm{E}=46-60 \mu \mathrm{m}, \mathrm{P} /$ $\mathrm{E}=1,02-1,71 \mu \mathrm{m})$, ámbito circular. Colpos largos varían entre 56 y $71 \mu \mathrm{m}$ de longitud. Exina tectada de 2,2-3,3 $\mu \mathrm{m}$ de espesor. Ornamentación estriada-escábrida.

MEB: Los colpos en vista polar son redondeados en sus extremos (Fig. 6.F) y la ornamentación es estriada (Fig. 6.G) y psilada hacia el apocolpium (Fig. 6.H).

Distribución geográfica en Chile: Región II

\section{N. miersii var. miersii Remy (Fig. 3.A-C)}

MO: Granos subprolatos $(\mathrm{P}=59-86 \mu \mathrm{m}, \mathrm{E}=49-74 \mu \mathrm{m}, \mathrm{P} /$ $\mathrm{E}=0,88-1,58 \mu \mathrm{m})$, ámbito circular. Colpos largos varían entre 58 y $81 \mu \mathrm{m}$ de longitud. Exina tectada de 1,9-3,1 $\mu \mathrm{m}$ de espesor. Ornamentación estriada.

MEB: La ornamentación es rugulada-estriada (Fig. 7.A).

Distribución geográfica en Chile: Regiones III, IV, V, Región Metropolitana de Santiago.

\section{N. pauciflora Remy (Fig. 3. D-F)}

MO: Granos subprolatos $(\mathrm{P}=64-81 \mu \mathrm{m}, \mathrm{E}=51-65 \mu \mathrm{m}, \mathrm{P} /$ $\mathrm{E}=1,02-1,54 \mu \mathrm{m})$, ámbito circular. Colpos largos varían entre 57 y $71 \mu \mathrm{m}$ de longitud. Exina tectada de 2,0-2,8 $\mu \mathrm{m}$ de espesor. Ornamentación estriada-escábrida.

MEB: Ornamentación estriada (Fig. 7.C) y psilada hacia el apocolpium (Fig. 7.D).

Distribución geográfica en Chile: Regiones III, IV.

\section{N. petunioides (Griseb) Millán (Fig. 1. I,I1)}

MO: Granos subprolatos $(\mathrm{P}=53-70 \mu \mathrm{m}, \mathrm{E}=38-60 \mu \mathrm{m}, \mathrm{P} /$ $\mathrm{E}=0,95-1,65 \mu \mathrm{m})$, ámbito circular o semiangular. Colpos largos varían entre 50 y $63 \mu \mathrm{m}$ de longitud. Exina tectada de 1,9-2,9 $\mu \mathrm{m}$ de espesor. Ornamentación estriada.

MEB: Ornamentación estriada, psilada hacia el apocolpium (Fig. 7.F); con magnificación 31.550x se observan perforaciones en la pared (Fig. 7.G).

Distribución geográfica: Regiones II, III

N. solanifolia Walp. (Fig. 4.A-C)

MO: Granos subprolatos $(\mathrm{P}=57-73 \mu \mathrm{m}, \mathrm{E}=38-66 \mu \mathrm{m}, \mathrm{P} /$ $\mathrm{E}=0,98-1,7 \mu \mathrm{m})$, ámbito circular.

Colpos largos varían entre 53 y $67 \mu \mathrm{m}$ de longitud. Exina tectada de 2,0-2,9 $\mu \mathrm{m}$ de espesor. Ornamentación estriada.

MEB: Ornamentación rugulada (Fig. 8.B) y psilada hacia el apocolpium (Fig. 7.C).

Distribución geográfica: Regiones I, II, III, IV

N. tabacum L. (Fig. 4.D-F)

MO: Granos prolatos $(\mathrm{P}=68-83 \mu \mathrm{m}, \mathrm{E}=48-61 \mu \mathrm{m}, \mathrm{P} / \mathrm{E}=$ 1,21-1,59 $\mu \mathrm{m})$, ámbito circular. La Colpos largos varían entre 50 y $71 \mu \mathrm{m}$ de longitud. Exina tectada de 2,0-2,8 $\mu \mathrm{m}$ de espesor. Ornamentación estriada a reticuladaheterobrocada.

MEB: Ornamentación estriada, psilada hacia el apocolpium (Fig. 8.D); con magnificación 26.980x se observan perforaciones en la pared (Fig. 8.E)

N. undulata Ruiz et Pav. (Fig. 4.G-I)

MO: Granos prolato-esferoidales $(\mathrm{P}=57-77 \mu \mathrm{m}, \mathrm{E}=46$ $66 \mu \mathrm{m}, \mathrm{P} / \mathrm{E}=0,9-1,5 \mu \mathrm{m})$, ámbito circular. Colpos largos varían entre 50 y $69 \mu \mathrm{m}$ de longitud. Exina tectada de 2,12,8 $\mu \mathrm{m}$ de espesor. Ornamentación estriada a reticuladaheterobrocada.

MEB: Ornamentación rugulada-estriado, con presencia de perforaciones (Fig. 8.H) y psilada hacia el apocolpium (Fig. 8.H).

Distribución geográfica: Región II.

Análisis estadístico de los granos de polen no calcinados

Las especies estudiadas son en general homogéneas desde un punto de vista palinológico; sin embargo, análisis estadísticos globales y también entre pares de especies reportaron diferencias significativas en la longitud de los colpos largos (Kruskall-Wallis: $\mathrm{H}_{11}=132,703$; $P<0,001$ ), en el diámetro ecuatorial, E (KruskallWallis: $\left.\mathrm{H}_{11}=137,264 ; P<0,001\right)$, en el diámetro polar, $\mathrm{P}$ (Kruskall-Wallis: $\mathrm{H}_{11}=123,751 ; P<0,001$ ) y en el espesor de la exina (ANOVA: $\mathrm{F}_{11,299}=7,367$; $P<0,001)$. La tabla 1 resume las características cuantitativas de los morfotipos polínicos estudiados. 


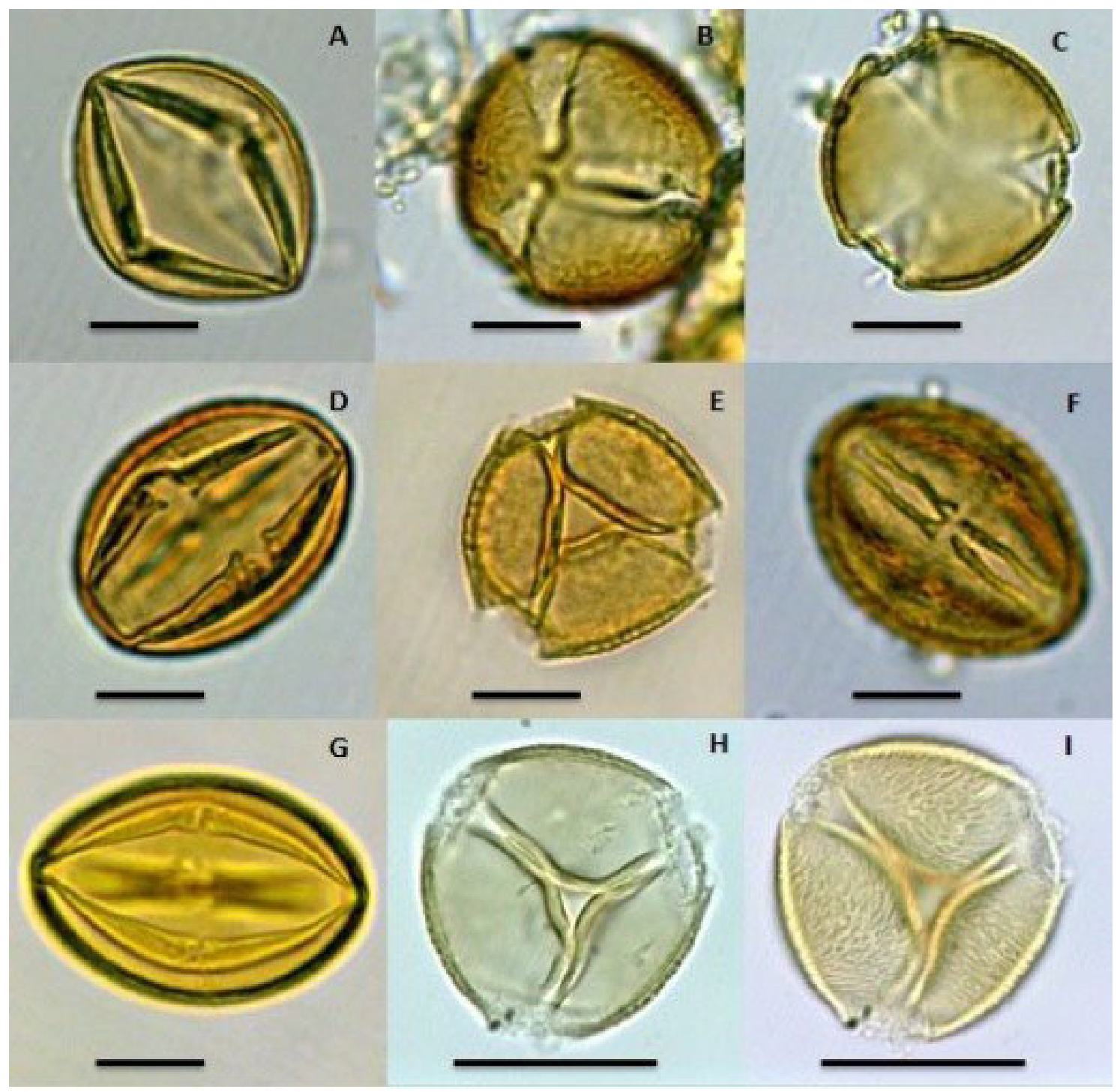

Fig. 1. Fotografías en microscopía óptica de polen de especies del género Nicotiana presentes en Chile. A-C, Nicotiana acuminata: A, vista ecuatorial (VE). B, vista polar (VP). C, detalle de la pared en VP. D-F, N. cordifolia: D, VE. E, VP. F, detalle de la pared y de un poro en VE. G-I, N. corymbosa: G, VE. H, VP. I, detalle de la pared en VP. Barra escala: $20 \mu \mathrm{m}, 400 x$ (excepto H,I: $10 \mu \mathrm{m}, 1000 x$ ).

Descripción palinológica de especies de tabaco calcinadas

Bajo MEB, los granos de polen calcinados de $N$. tabacum evidenciaron daño y alteraciones crecientes en la ornamentación de la pared y en la forma del grano a medida que se aumentó la temperatura (Figs. 9 y 10)

\section{N. tabacum calcinado a $150^{\circ} \mathrm{C}$}

La ornamentación se mantuvo completa y sin alteraciones evidentes con respecto a un grano sin calcinar (Fig. 9A).

\section{N. tabacum calcinado a $165^{\circ} \mathrm{C}$}

Las estriaciones de la pared se tornaron levemente pronunciadas y globosas (Fig. 9.B.1) y en algunos casos la pared estaba quebrada (Fig. 9.B.2). 


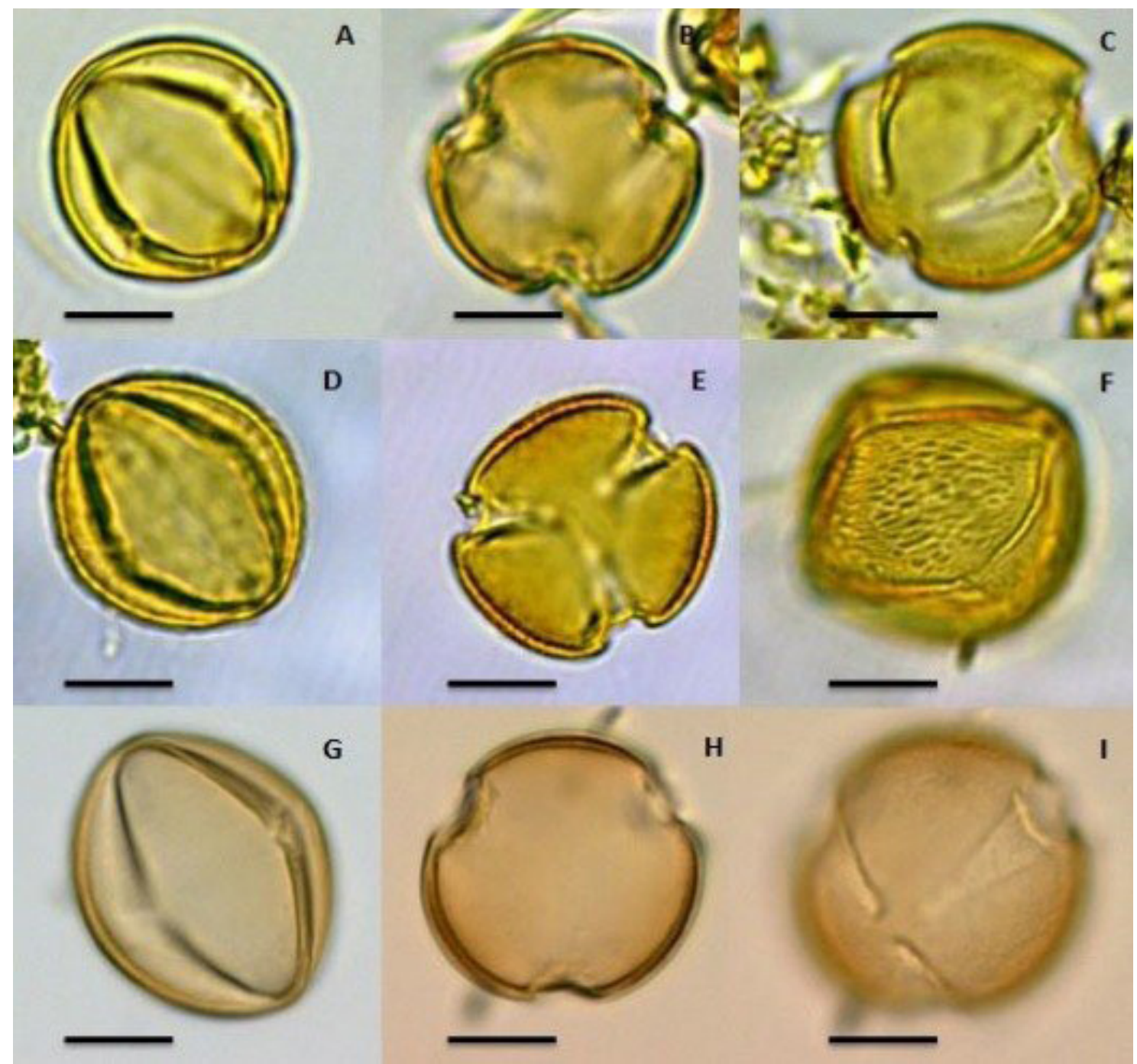

Fig. 2. Fotografías en microscopia óptica de polen de especies del género Nicotiana presentes en Chile. A-C, Nicotiana glauca: A, vista ecuatorial (VE). B, vista polar (VP). C, detalle de la pared en VP. D-F, N. linearis: D, VE. E, VP. F, detalle de la pared en VE. G-I, N. longibracteata: G, VE. H, VP. I, detalle de la pared en VP. Barra escala: $20 \mu \mathrm{m}, 400 x$ (excepto C: $10 \mu \mathrm{m}, 1000 x$ ).

\section{N. tabacum calcinado a $185^{\circ} \mathrm{C}$}

Los colpos se observaron abiertos y profundos (Fig. 9.C.1), las estriaciones de la pared más globosas que a $165^{\circ} \mathrm{C}$, similares a verrugas (Fig. 9.C.2) y ya fue posible encontrar granos completamente deformes (Fig. 9C.3).

\section{N. tabacum calcinado a $205^{\circ} \mathrm{C}$}

Los colpos se observaron con los bordes quebrados y separados (Fig. 9.D.1), las estriaciones de la ornamentación muy pronunciadas y verrucoides y en algunos granos destruidas.

\section{N. tabacum calcinado a $230^{\circ} \mathrm{C}$}

Los colpos se encontraron deformados, sus bordes separados, el interior la pared apareció destruida (Fig. 10E.1) y las estriaciones en ella muy pronunciadas (Fig. 10E.2). 


\section{K. Collao-Alvarado et al. - Polen de especies de Nicotiana en Chile}

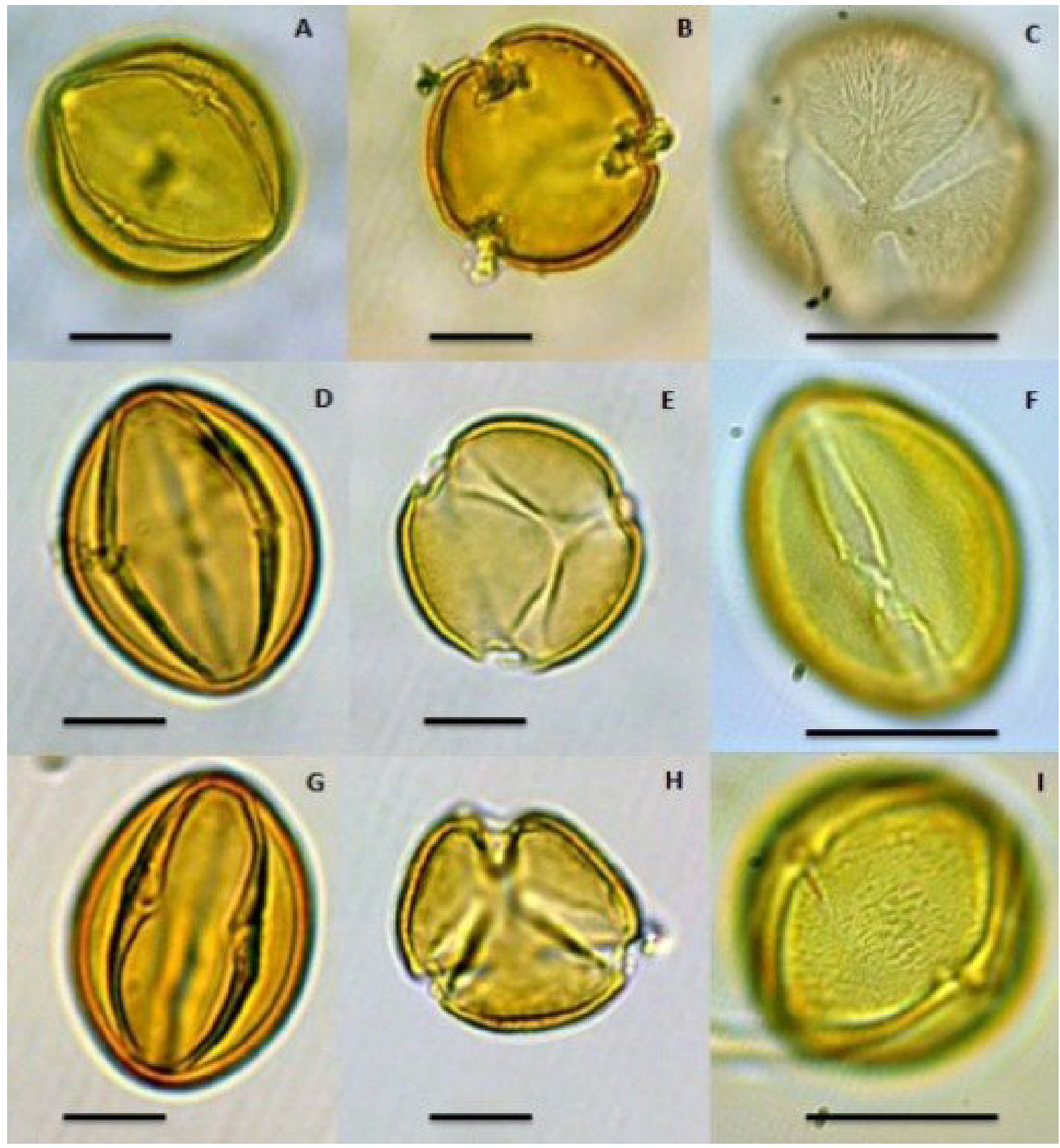

Fig. 3. Fotografías en microscopia óptica de polen de especies del género Nicotiana presentes en Chile. A-C, Nicotiana miersii var. miersii: A, vista ecuatorial (VE). B, vista polar (VP). C, detalle de la pared en VP. D-F, N. pauciflora: D, VE. E, VP. F, detalle de la pared en VE. G-I, N.petunioides: G, VE. H, VP. I, detalle de la pared en VE. Barra escala: $20 \mu \mathrm{m}, 400 x$ (excepto C,F,I: $10 \mu \mathrm{m}, 1000 x$ ).

\section{N. tabacum calcinado a $290^{\circ} \mathrm{C}$}

La mayoría de los granos mostraron la ornamentación de la pared destruida, las estriaciones deformadas y la estructura verrucoide ausente (Fig. 10F.1), y tanto los granos de polen como los colpos aparecieron deformados (Fig. 10F.2). A $320^{\circ} \mathrm{C}$ los granos de polen se convirtieron en ceniza. 
Bol. Soc. Argent. Bot. 51 (1) 2016

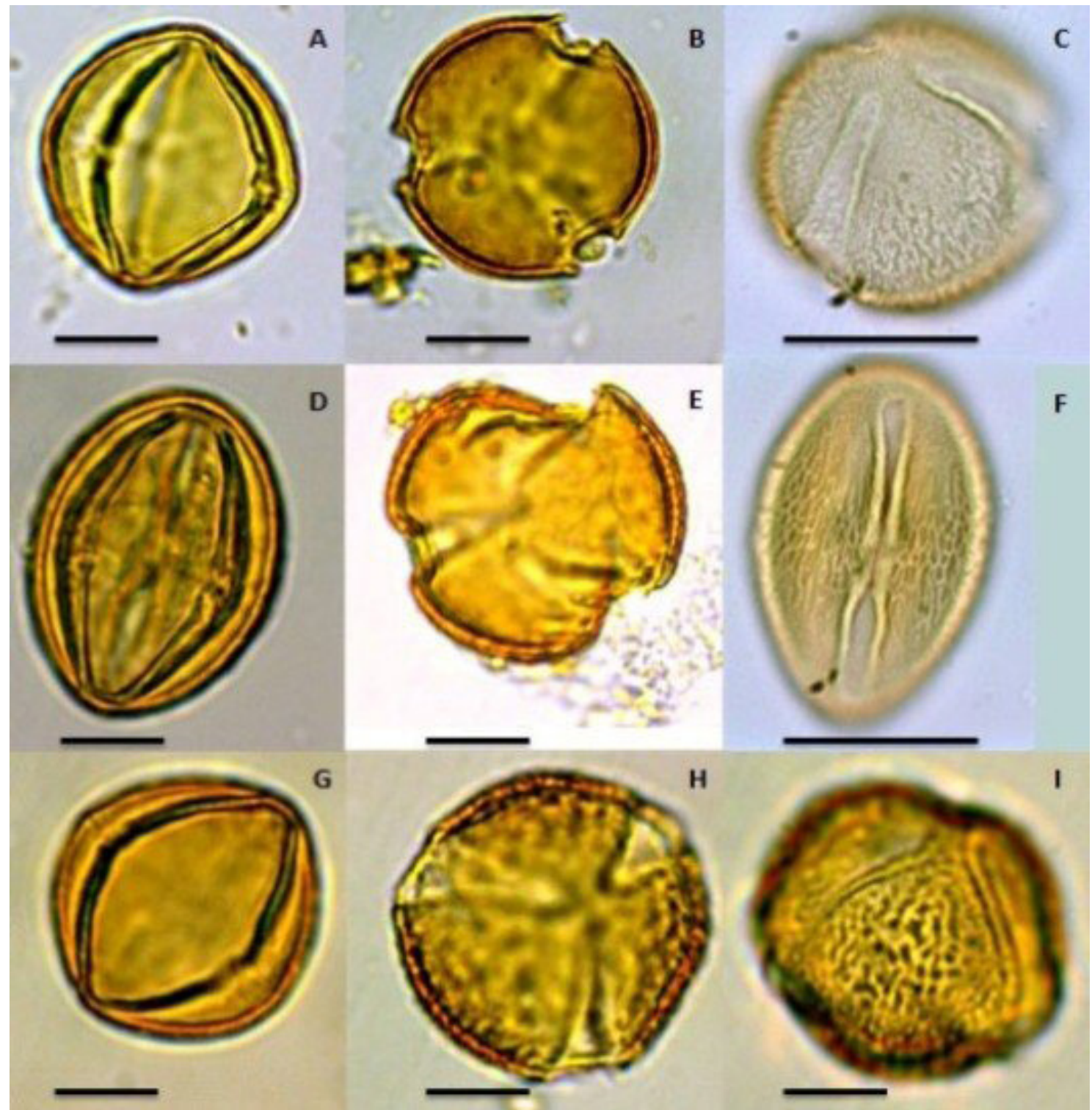

Fig. 4. Fotografías en microscopia óptica de polen de especies del género Nicotiana presentes en Chile. A-C, Nicotiana solanifolia: A, vista ecuatorial (VE). B, vista polar (VP). C, detalle de la pared en VP. D-F, N. tabacum: D, VE. E, VP. F, detalle de la pared en VE. G-I, N.undulata: G, VE. H, VP. I, detalle de la pared en VP. Barra escala: $20 \mu \mathrm{m}, 400 x$ (excepto C,F: $10 \mu \mathrm{m}, 1000 x$ ).

\section{Discusión}

Las especies de Nicotiana en Chile presentan granos isopolares, tectados, 3-colporados o 3-zonocolporados; el ámbito en general es circular y la ornamentación es estriada a estriada-rugulada. En todas las especies, la ornamentación es psilada en los polos. Cabe destacar que bajo MEB y con magnificación mayor que 20.000x, en algunas especies de Nicotiana la ornamentación se observa verrucoide y la exina presenta perforaciones en la pared.

Gish (2000) describió, usando material de herbario, el polen de 14 géneros de Solanaceae 


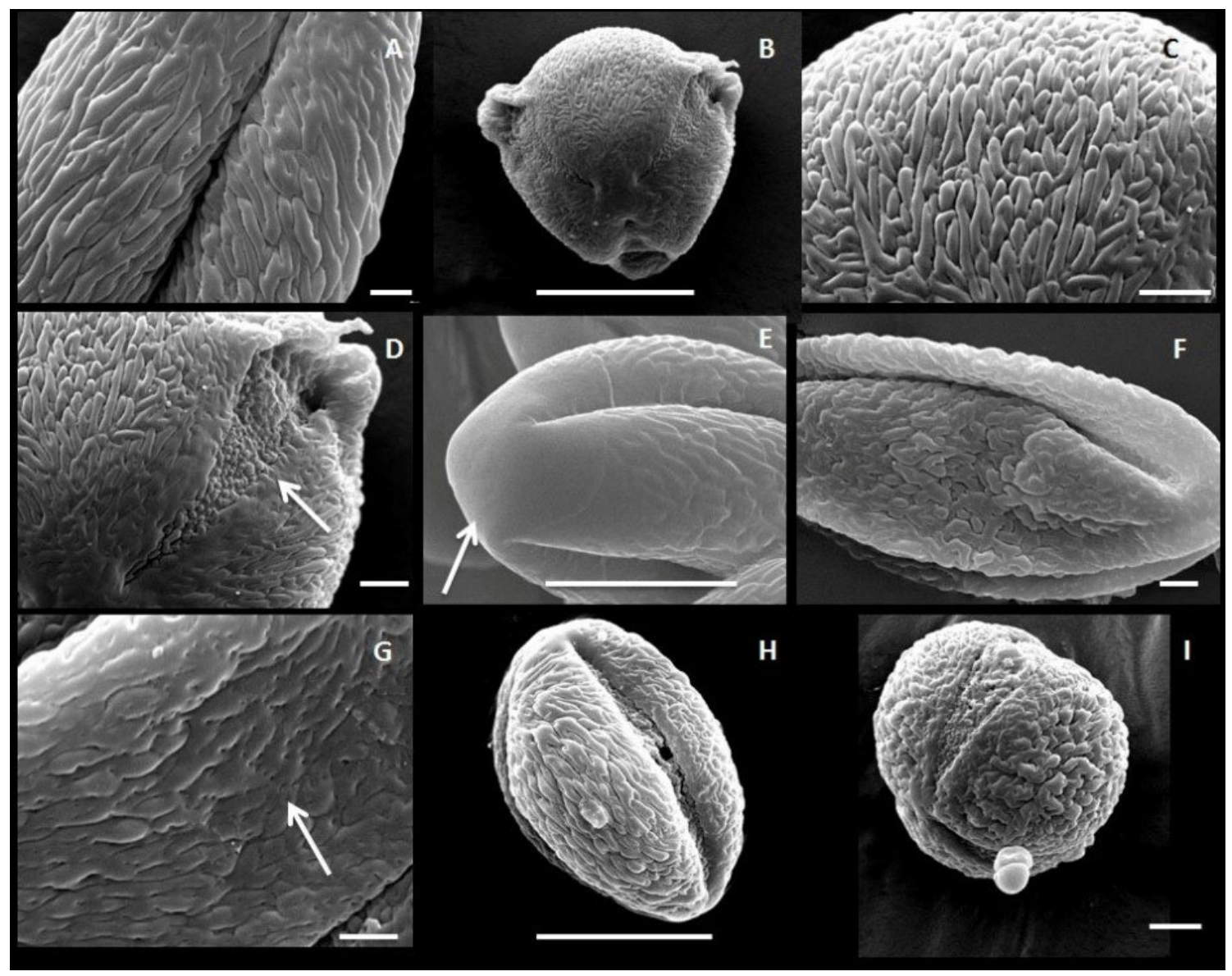

Fig. 5. Fotografías en microscopía electrónica de barrido de polen de especies del género Nicotiana presentes en Chile. A-E, Nicotiana acuminata: A, vista ecuatorial (VE) (41.000x). B, vista polar (VP) (18.000x). C, detalle pared en VP (65.400x). D, detalle de gránulos en el interior de un colpo (flecha) en VP (45.600x). E, VP (20.990x). F-G, N. cordifolia: F, VE (35.600x). G, detalle de pared (67.000x). H-I, N. corymbosa: I, detalle de pared y colpo en VE (20.000x). H, VE (18.000x). En E y H la flecha indica la ornamentación psilada del apocolpium (VP y VE, respectivamente). Barra escala: $1 \mu \mathrm{m}$ (excepto B, E, H: $10 \mu \mathrm{m})$.

presentes en Norteamérica; dentro del género Nicotiana, describió el polen de $N$. tabacum, $N$. acuminata y $N$. glauca. En el caso de N. tabacum, señaló un cociente $\mathrm{P} / \mathrm{E}$ entre 1 y 1,2 , exina estriada a reticulada y aberturas 3-colporadas y 4-colporadas. En el presente estudio, los granos de $N$. tabacum mostraron un cociente $\mathrm{P} / \mathrm{E}$ de 1,4 \pm 0,02 y no se observó dimorfismo, solo se encontraron granos 3-colporados; sin embargo, ambas descripciones corresponden a granos subprolatos según la equivalencia descrita por Erdtman (1952). Según Gish (2000), una de las posibles explicaciones del carácter 4-colporado del polen de $N$. tabacum radicaría en la incompleta división celular de la microspora durante la meiosis que habría provocado una formación fallida de la tétrada y en consecuencia un grano inviable, característica que se ha observado en especies de Nicotiana domesticadas como la antes mencionada y en $N$. rustica. Para el polen de N. acuminata, Gish (2000) describió un valor de P/E de 1,1, forma subesferoidal y ornamentación estriada reticulada; en nuestro estudio, el polen de $N$. acuminata mostró un cociente $\mathrm{P} / \mathrm{E}$ levemente mayor $(1,2 \pm 0,02)$, con forma prolato-esferoidal y ornamentación sin 
Bol. Soc. Argent. Bot. 51 (1) 2016

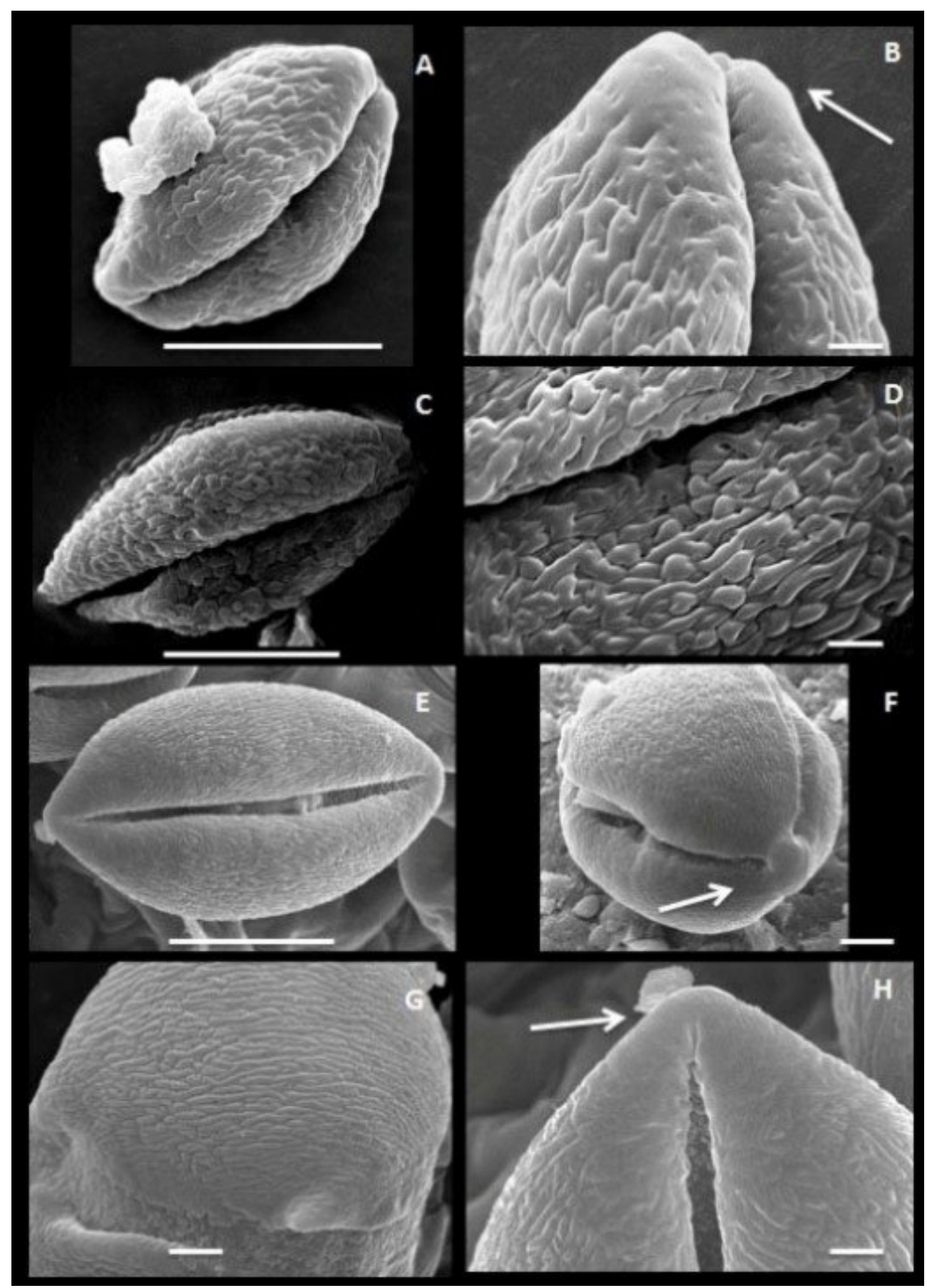

Fig. 6. Fotografías en microscopía electrónica de barrido de polen de especies del género Nicotiana presentes en Chile. A-B, Nicotiana glauca: A, vista ecuatorial (VE) y detalle de pared (22.859x). B, vista ecuatorial (VE) (50.690x). C-D, N. linearis: C, VE (43.830x). D, detalle de pared en VE (43.830x). E-H, N. longibracteata: E, VE (17.290x). F, detalle de colpo en VP (flecha) (33.630x). G, detalle de pared (43.800x). H, detalle del extremo polar en VE (40.830x). En B y H la flecha indica la ornamentación psilada del apocolpium, ambos en VE. Barra escala: $1 \mu \mathrm{m}$. (Excepto A: $10 \mu \mathrm{m}$ ) 
K. Collao-Alvarado et al. - Polen de especies de Nicotiana en Chile

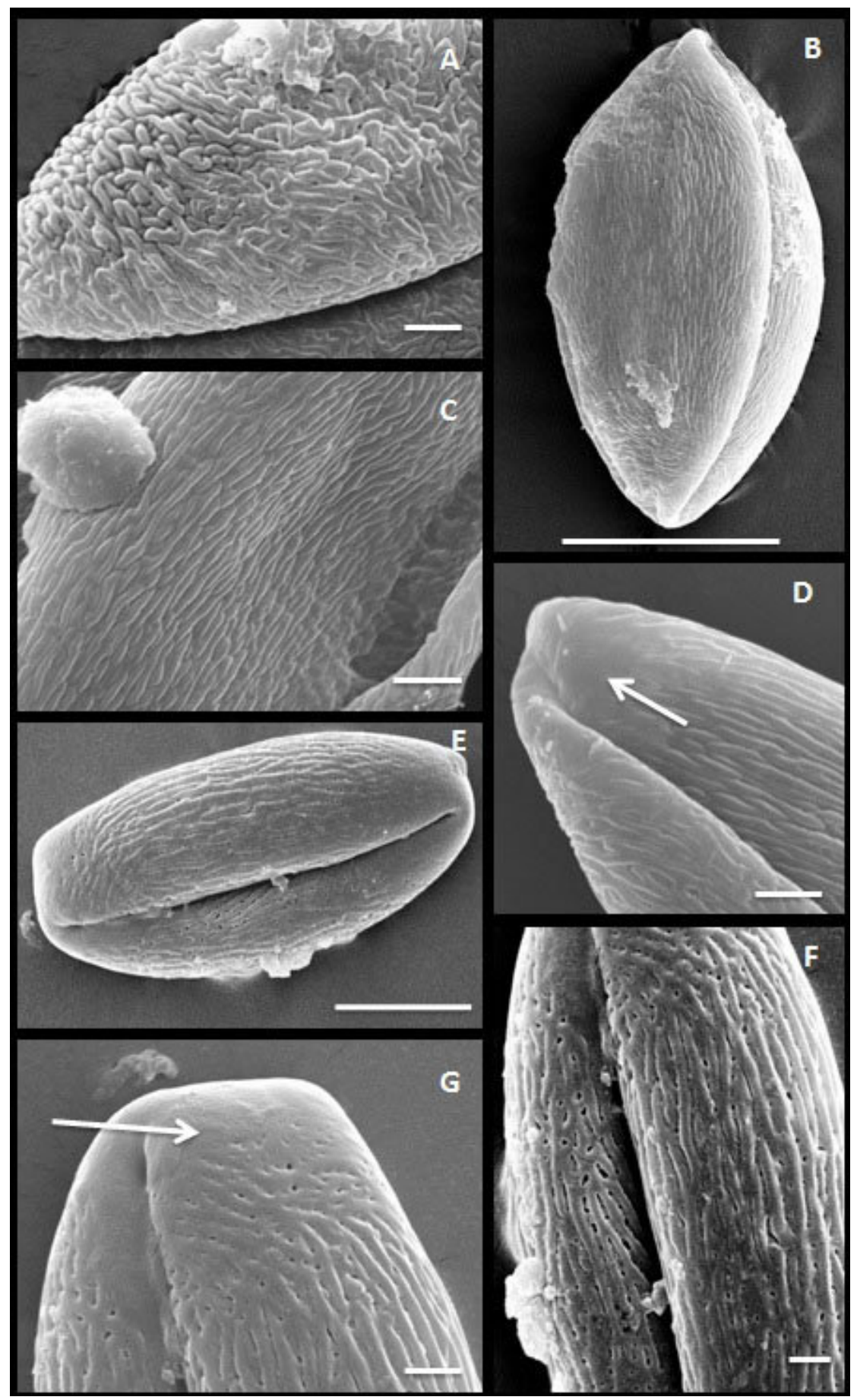

Fig. 7. Fotografías en microscopía electrónica de barrido de polen de especies del género Nicotiana presentes en Chile. A, Nicotiana miersii var. miersii: A, vista ecuatorial (VE) y detalle de pared (47.640x). B-D, N. pauciflora: B, VE (21.400x). C, detalle de pared en VE (56.480x). D, VE (59.700x). E-G, N.petunioides: E, VE (13.360x). F, detalle de pared en VE (flecha) (31.550x). G, detalle del extremo polar en VE (44.620x). En D y $\mathbf{G}$ la flecha indica la ornamentación psilada de apocolpium ambos en VE. Barra escala: 1 um. 
Bol. Soc. Argent. Bot. 51 (1) 2016

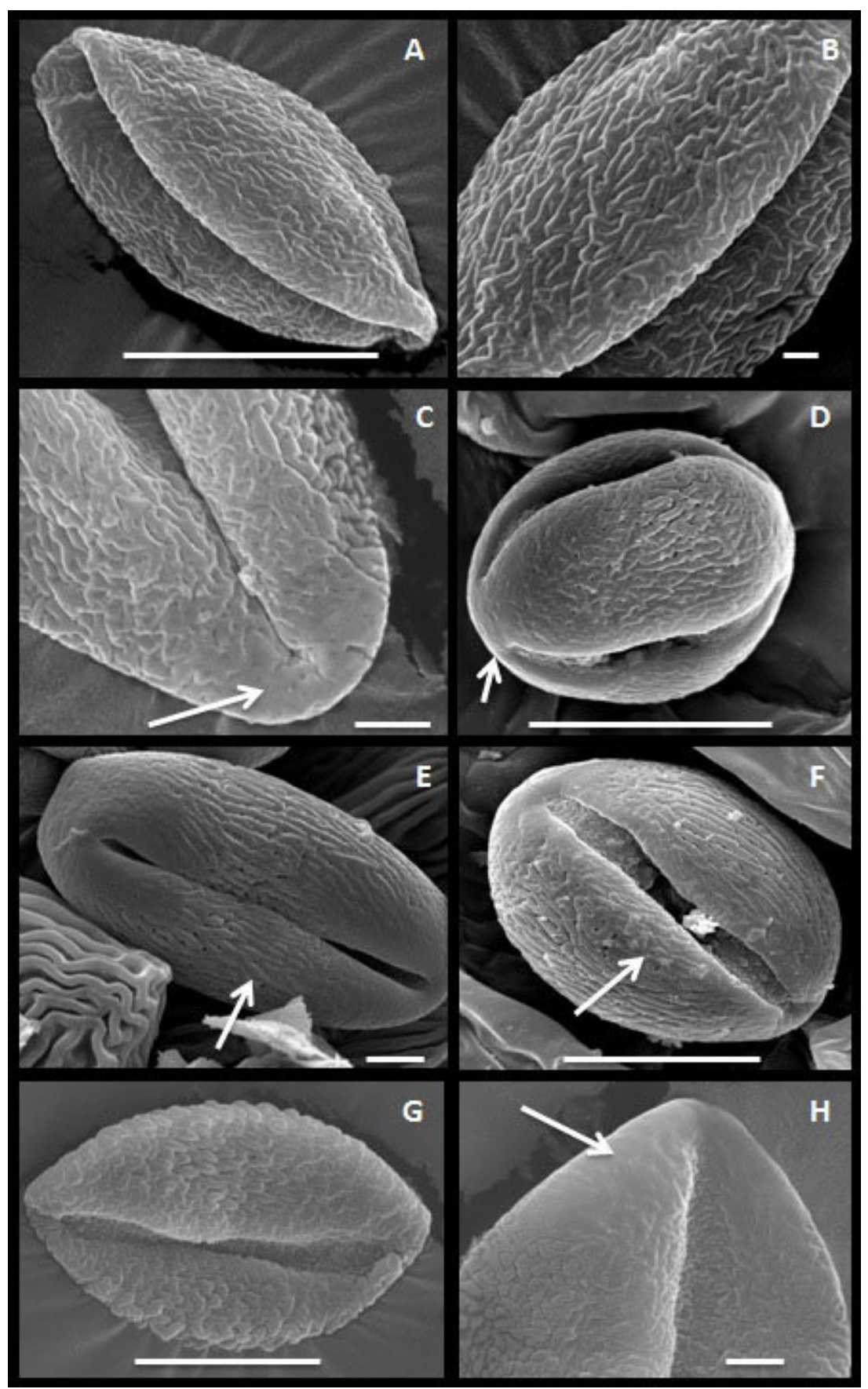

Fig. 8. Fotografías en microscopía electrónica de barrido de polen de especies del género Nicotiana presentes en Chile. A-C, Nicotiana solanifolia: A, vista ecuatorial (VE) (27.740x). B, detalle de pared en VE (27.740x). C, detalle del extremo polar en VE; la flecha indica la ornamentación psilada del apocolpium (61.760x). D-F, N. tabacum: D, VE y detalle de apocolpium (23.700x). E, detalle de pared en VE (26980x). F, detalle de colpo y poro (flecha) en VE (18.820x). G-H, N.undulata: G, grano en VE (17.320x). H, detalles de la ornamentación estriada (flecha inferior) y psilada del apocolpium (flecha superior) en VE (48.980x). Barra escala: $1 \mu \mathrm{m}$. 


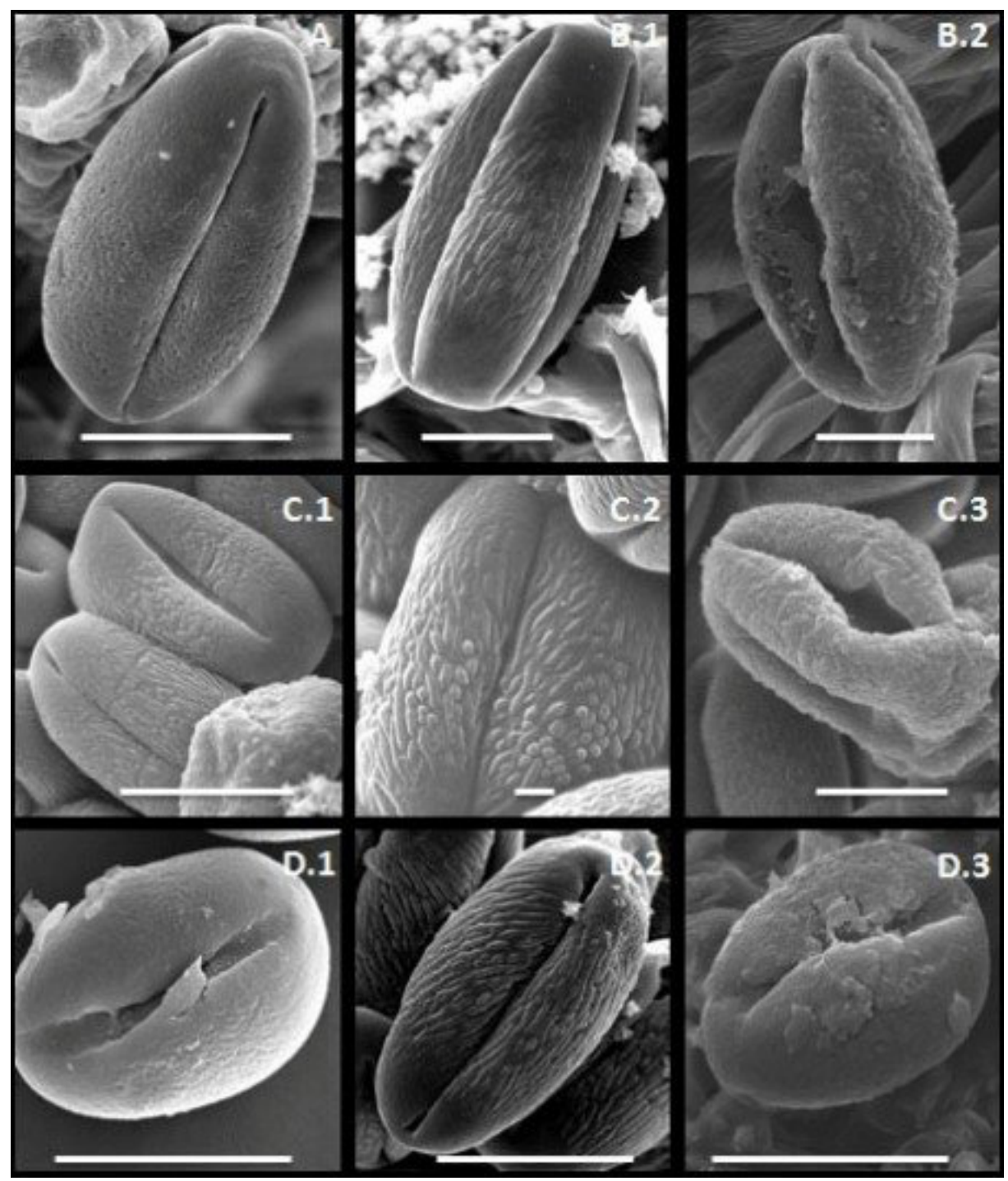

Fig. 9. Fotografías en microscopía electrónica de barrido de polen de Nicotiana tabacum calcinado a diferentes temperaturas. A, $150^{\circ} \mathrm{C}(17.670 \mathrm{x})$. B, $165^{\circ} \mathrm{C}$, B.1 (10.960X), B.2 (8.930x). C, $185^{\circ} \mathrm{C}$, C.1 (20.000x), C.2 (14.760x), C.3 (10.440x). D, $205^{\circ}$ C, D.1 (26.420x), D.2 (16.040x), D.3 (19.620X). Barra escala: $10 \mu \mathrm{m}$, excepto C.2: $1 \mu \mathrm{m}$.

presencia de retículos, sólo estriaciones y sectores escábridos en el grano. Finalmente, Gish (2000) describió el polen de $N$. glauca con valores de $\mathrm{P} / \mathrm{E}$ entre 1,1 y 1,7, forma prolato-subesferoidal, colpos largos y poros marcados. En el presente estudio la forma del polen de $N$. glauca fue subprolato, y el valor de $\mathrm{P} / \mathrm{E}(1,43 \pm 0,03)$ se encontró dentro del rango antes descrito, aunque los poros fueron débilmente notorios, tal como lo descrito por Heusser (1971). Por otra parte, Heusser (1971) describe el polen de $N$. glauca con presencia de colpos agudos y ligeramente redondeados y Polo \& Diez (1986) la describe con granos de tamaño pequeño, elípticos en vista ecuatorial, y valores de $\mathrm{P} / \mathrm{E}$ entre 0,85 y 1,35 , similares a los observados en el presente trabajo (Fig. 2C). 
Bol. Soc. Argent. Bot. 51 (1) 2016

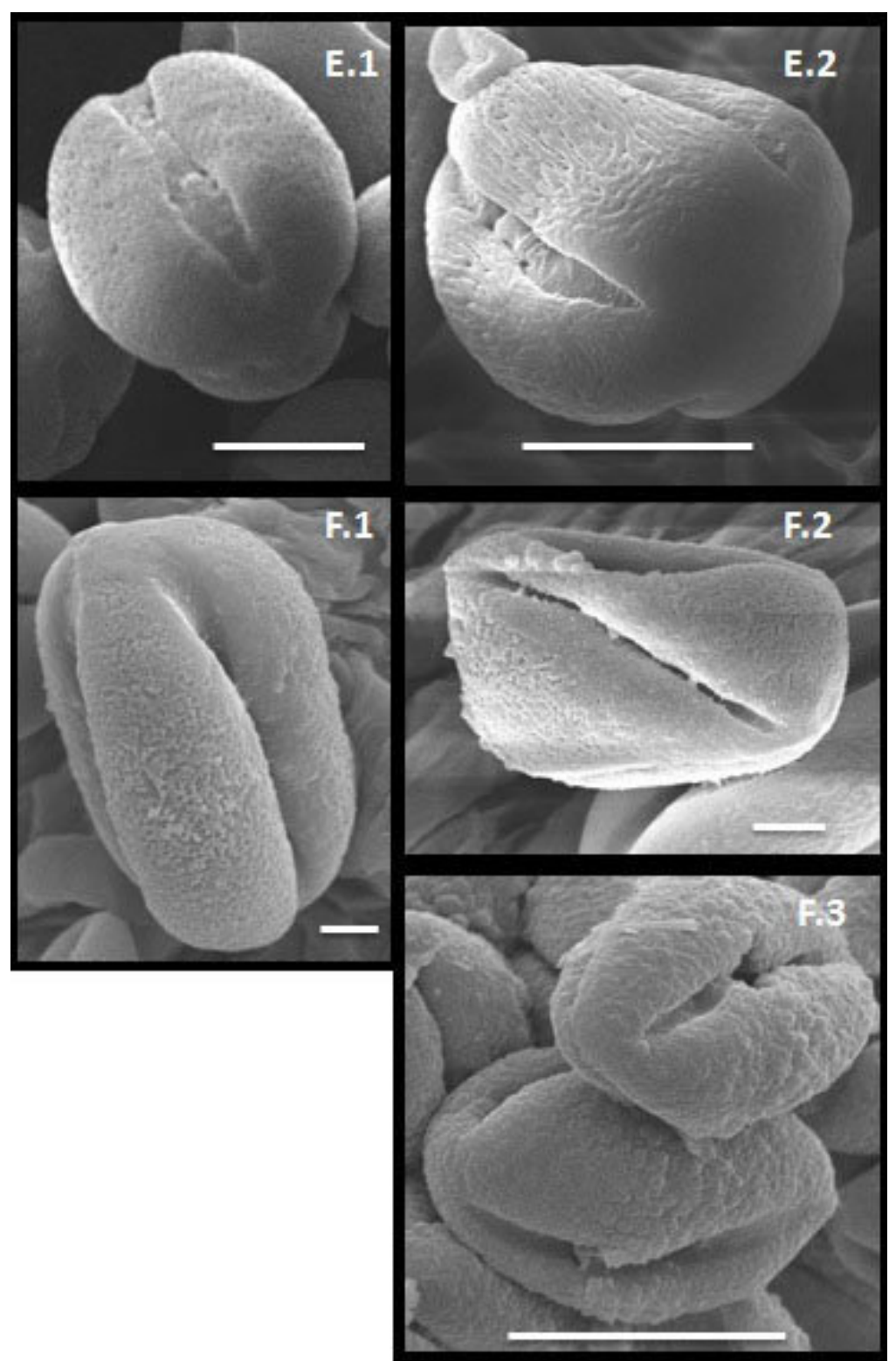

Fig. 10. Fotografías en microscopía electrónica de barrido de polen de Nicotiana tabacum calcinado a diferentes temperaturas. E. $230^{\circ} \mathrm{C}$, E.1 (17.320x), E.2 (11.020x). F. $290^{\circ} \mathrm{C}$, F.1 (34.380x), F.2 (34.490), F.3 (20.020x). Barra escala: $10 \mu \mathrm{m}$, excepto F.1 y F.2: $1 \mu \mathrm{m}$.

Las comparaciones cualitativas descritas en los párrafos anteriores sugieren que las especies de Nicotiana presentes en Chile tienen en general características cualitativas similares. Por otra parte y aún cuando los parámetros cuantitativos muestran diferencias significativas entre algunas especies (Tabla 1), estas mediciones no pueden ser utilizadas para identificar especies pues la magnitud de las varianzas no lo permiten. En consecuencia, las mediciones realizadas sobre un eventual grano de polen que se encuentre en un yacimiento o en determinados artefactos no 


\section{K. Collao-Alvarado et al. - Polen de especies de Nicotiana en Chile}

Tabla 1. Características morfológicas cuantitativas de los granos de polen de 12 especies de Nicotiana. $\mathrm{P}=$ diámetro polar y $\mathrm{E}=$ diámetro ecuatorial, ambos en vista ecuatorial. Se muestran promedios \pm desviación estándar y la significancia de las diferencias entre especies (los parámetros muestran diferencias significativas entre especies si las combinaciones de letras en la columna correspondiente son distintas).

\begin{tabular}{|c|c|c|c|c|c|}
\hline \multirow{2}{*}{ Especie } & \multirow{2}{*}{$\begin{array}{c}\text { Forma } \\
\text { P/E }\end{array}$} & \multicolumn{2}{|c|}{ Tamaño $(\mu \mathrm{m})$} & \multirow{2}{*}{$\begin{array}{l}\text { Espesor exina } \\
\qquad(\mu \mathrm{m})\end{array}$} & \multirow{2}{*}{$\begin{array}{c}\text { Colpos } \\
\text { largos } \\
(\mu \mathrm{m})\end{array}$} \\
\hline & & $\mathbf{P}$ & E & & \\
\hline \multirow{2}{*}{ N. acuminata } & $1,20 \pm 0,11$ & $76,6 \pm 4,31$ & $63,8 \pm 5,0$ & $2,1 \pm 0,23$ & $68,12 \pm 3,81$ \\
\hline & be & a & a & a & ac \\
\hline \multirow{2}{*}{ N. cordifolia } & $1,28 \pm 0,14$ & $69,4 \pm 5,34$ & $54,4 \pm 3,21$ & $2,29 \pm 0,28$ & $56,86 \pm 4,26$ \\
\hline & acdefg & bdef & bfg & ace & be \\
\hline \multirow{2}{*}{ N. corymbosa } & $1,29 \pm 0,19$ & $75,3 \pm 4,5$ & $59,03 \pm 7,24$ & $2,6 \pm 0,29$ & $65,67 \pm 6,73$ \\
\hline & acefgh & ace & acef & bd & ac \\
\hline \multirow{2}{*}{ N. glauca } & $1,43 \pm 0,19$ & $66,9 \pm 7,85$ & $46,8 \pm 4,02$ & $2,3 \pm 0,26$ & $57,16 \pm 6,73$ \\
\hline & acd & bfgh & g & ace & bde \\
\hline \multirow{2}{*}{ N. linearis } & $1,14 \pm 0,16$ & $72,8 \pm 8,1$ & $64,5 \pm 8,5$ & $2,5 \pm 0,35$ & $67,70 \pm 6,64$ \\
\hline & be & aceh & ad & be & ac \\
\hline \multirow{2}{*}{ N. longibracteata } & $1,24 \pm 0,18$ & $68,4 \pm 6,96$ & $55,3 \pm 4,1$ & $2,7 \pm 0,31$ & $64,49 \pm 4,09$ \\
\hline & bde & bfgh & bc & $b$ & acf \\
\hline \multirow{2}{*}{ N. miersii var. miersii } & $1,18 \pm 0,16$ & $75,9 \pm 7,2$ & $64,4 \pm 6,4$ & $2,5 \pm 0,31$ & $69,77 \pm 6,19$ \\
\hline & bg & ace & a & be & a \\
\hline \multirow{2}{*}{ N. pauciflora } & $1,28 \pm 0,13$ & $73,8 \pm 5,31$ & $57,8 \pm 4,0$ & $2,4 \pm 0,24$ & $63,36 \pm 4,04$ \\
\hline & acefgh & acf & acef & acde & acdf \\
\hline \multirow{2}{*}{ N. petunioides } & $1,14 \pm 0,14$ & $61,8 \pm 4,8$ & $54,3 \pm 4,0$ & $2,4 \pm 0,27$ & $54,35 \pm 4,54$ \\
\hline & bf & $b$ & bf & be & $b$ \\
\hline \multirow{2}{*}{ N. solanifolia } & $1,22 \pm 0,20$ & $67,2 \pm 4,55$ & $56,1 \pm 7,74$ & $2,45 \pm 0,24$ & $59,52 \pm 4,83$ \\
\hline & bh & bgh & bcd & be & bf \\
\hline \multirow{2}{*}{ N. tabacum } & $1,4 \pm 0,10$ & $75,8 \pm 4,6$ & $54,0 \pm 3,57$ & $2,47 \pm 0,25$ & $62,35 \pm 4,77$ \\
\hline & a & acd & befg & be & cef \\
\hline \multirow{2}{*}{ N. undulata } & $1,11 \pm 0,14$ & $63,8 \pm 5,9$ & $57,5 \pm 4,5$ & $2,4 \pm 0,19$ & $62,20 \pm 5,90$ \\
\hline & $\mathrm{b}$ & $\mathrm{b}$ & acef & ace & cef \\
\hline
\end{tabular}

permitirán su identificación específica, aunque sí se podrá identificar, con base en las fotos incluidas en el presente trabajo, como perteneciente al género Nicotiana. En efecto, el polen como biomarcador al nivel de familia y eventualmente al nivel de género ha sido utilizado con éxito en contextos tanto actuales como arqueológicos (Bryant et al., 2012; Buosi et al., 2013; Winter, 2000). La posibilidad de discriminar entre el polen de tabaco y el de otros grupos taxonómicos tiene importantes implicancias arqueológicas, ya que la presencia de microrresiduos de tabaco en artefactos tales como pipas, micromorteros, ceramios, tabletas y tubos de inhalación, entre otros, constituye una evidencia de actividades culturales relacionadas a ritualidad, intercambio de bienes y congregación social, que dependiendo de la región geográfica y los datos arqueológicos o etnográficos disponibles posibilitarían la reconstrucción del contexto histórico y cultural del hallazgo.

Por otro lado, investigaciones recientes demostraron que los granos de polen recuperados desde material arqueológico calcinado puede ser contabilizado y determinado con éxito al nivel de familia y/o género (Bryant et al., 2012). Nuestros datos de calcinación experimental de granos de polen indican que a las temperaturas que pueden medirse lejos del punto de ignición del material fumado y cerca de las paredes de una pipa (ca. $150^{\circ} \mathrm{C}$; Ermala \& Holsti 1956), los granos de polen no sufren alteraciones significativas. Las 
alteraciones son mayores a temperaturas más altas, de modo que en un residuo del interior de una pipa, ya sea del hornillo, tubo o boquilla, es posible encontrar granos de polen con distintos grados de alteración. En todo caso, nuestros estudios muestran que los granos alterados serán distinguibles de los intactos y podrán utilizarse solo estos últimos en las mediciones orientadas a la identificación.

En conclusión, las características morfológicas cualitativas y cuantitativas de los granos de polen de las especies de Nicotiana son demasiado homogéneas como para ser usadas en la distinción entre una u otra especie del género. Considerando las interacciones descritas entre las poblaciones prehispánicas de Chile y Argentina en un amplio rango latitudinal y temporal (Niemeyer et al., 1989; Lagiglia, 1997; Sanhueza et al., 2004; Cornejo \& Sanhueza, 2011; Stovel, 2008; Niemeyer, 2013; Niemeyer et al., 2015), sugerimos la importancia de realizar un estudio análogo con el polen de otras especies de Nicotiana que crecen en Argentina.

\section{Agradecimientos}

Agradecemos a Alicia Marticorena, curadora del Herbario de la Universidad de Concepción y Rosy Montero por facilitar el acceso a las muestras de Nicotiana, a Mauricio Mack del equipo de Microscopia Electrónica de la Facultad de Ciencias Sociales de la Universidad de Chile por su ayuda con las imágenes de microscopía electrónica y a dos evaluadores anónimos por sus generosas y acuciosas sugerencias. Este trabajo recibió el apoyo financiero del Proyecto FONDECYT 1121097 "Complejos fumatorios del periodo Alfarero Temprano en Chile semiárido y centro sur: un estudio multidisciplinario".

\section{Bibliografía}

AGASHE, S. N. \& E. CAULTON. 2009. Pollen and spores: aplications with special emphasis on aerobiology and allergy. Science Publishers, Enfield, $\mathrm{NH}$.

ANDREONI, D., R. SPANO \& V. LEMA. 2012. Nota sobre evidencias de uso de plantas en el sitio Soria
2 a partir del análisis microscópico del contenido de pipas. Arqueología 18: 235-243.

BELMAR, C., X. ALBORNOZ, S. ALFARO, F. MENESES, C. CARRASCO, L. QUIROZ, M. P. BABOT \& M. T. PLANELlA. 2015. Reconstruyendo las prácticas fumatorias del sitio La Granja (130 - 1000 D.C., valle del río Cachapoal, VI Región, Chile central), a partir de los microfósiles. Chungara, en prensa.

BRYANT, V. M., S. M. KAMPBELL \& J. L. HALL. 2012. Tobacco pollen: archaeological and forensic applications. Palynology 36: 208-223.

BUOSI, C., P. PITTAU, M. DEL RIO, D. MUREDDU \& M. C. LOCCI. 2013. A palynological investigation of funerary contents from Roman Imperial age necropolis in Sardinia (Italy). Palynology 37: 130-142.

CAPPARELLI,A., M. L. POCHETTINO, D. ANDREONI \& R. ITURRIZA. 2006. Differences between written and archaeological record: The case of plant micro remains recovered at a Northwestern Argentinean Pipe. Proceedings of the IVth International Congress of Ethnobotany (ICEB 2005): 397-406.

CORNEJO, L. \& L. SANHUEZA. 2011. Caminos que cruzan la Cordillera: el rol del Paso del Maipo en la ocupación de la Cordillera en Chile Central. Revista Chilena de Antropología № 23: 101-122

DONALDSON, M. P. \& W. E. STEPHENS. 2010. Environmental pollen trapped by tobacco leaf as indicators of the provenance of counterfeit cigarette products: a preliminary investigation and test of concept. J. Forensic Sci. 55: 738-741.

EERKENS, J., S. TUSHINGHAM, K. LENTZ, J. BLAKE, D. ARDURA, M. PALAZOGLU \& O. FIEHN. 2012. GC/MS analysis of residues reveals nicotine in two late prehistoric pipes from CAALA-554. SCA Proceedings 26: 212-219.

ECHEVERRÍA, J. \& H. M. NIEMEYER. 2013. Nicotine in the hair of mummies from San Pedro de Atacama (Northern Chile). J. Archaeol. Sci. 40: 3561-3568.

ECHEVERRÍA, J., M. T. PLANELLA \& H. M. NIEMEYER. 2014. Nicotine in residues of smoking pipes and other artifacts of the smoking complex from an Early Ceramic Period archaeological site in central Chile. J. Archaeol. Sci. 44: 55-60.

ERMALA, P. \& L. R. HOLSTI. 1956. On the burning temperatures of tobacco. Cancer Res. 16: 490-495.

ERDTMAN, G. 1952. Pollen morphology and plant taxonomy. Angiosperm (an introduction to palynology). En: TRIGO PÉREZ, M. D. M., MELGAR CABALLERO M. , GARCÍA SÁNCHEZ J., RECIO CRIADO M., DOCAMPO FERNÁNDEZ S. \& B. CABEZUDO ARTEDO (eds.), Polen en la Atmósfera de Vélez-Málaga, pp. 13-17, Concejalía del Medio Ambiente, Ayuntamiento de VélezMálaga, Almqvist \& Wiksell, Stockholm. 


\section{K. Collao-Alvarado et al. - Polen de especies de Nicotiana en Chile}

GISH, J. W. 2000. Morphological distinctiveness of Nicotiana pollen and the potential for identifying prehistoric Southwestern tobacco use through pollen analysis. En: J.C. WINTER (ed.), Tobacco Use by Native North Americans, pp. 223-261. Oklahoma: Norman, University of Oklahoma Press.

GOODSPEED, T. H. 1954. The Genus Nicotiana. Origins, Relationships and Evolution of its Species in the Light of their Distribution, Morphology and Cytogenetics. Chronica Botanica Company, Waltham.

HEUSSER, A. 1971. Pollen and Spores of Chile. Modern Types of the Pteridophita, Gymnospermaea and Angiospermae. Arizona, The University of Arizona Press.

LAGIGLIA, H. 1997. Arqueología de cazadores recolectores cordilleranos de altura. ICN. Ediciones Ciencia y Arte. San Rafael, Argentina.

LATCHAM, R. 1924. La organización socialy las creencias religiosas de los antiguos Araucanos. Publicaciones del Museo de Etnología y Antropología 3: 245-268.

MARTICORENA, C. \& M. QUEZADA. 1985. Catálogo de la flora vascular de Chile. Gayana 42: 1-157.

MUSSHOFF, F., W. ROSENDAHL \& B. MADEA. 2009. Determination of nicotine in hair samples of pre-Columbian mummies. Forensic Sci. Int. 185: 84-88.

NIEMEYER, H., G. CASTILLO \& M. CERVELLINO.1989.Los primeros ceramistas del Norte Chico. Complejo El Molle (0-800 d.C.). En: HIDALGO J., SCHIAPPACASSE V. , NIEMEYER H. , ALDUNATE C. \& I. SOLIMANO (eds.), Prehistoria. Culturas de Chile (eds.), pp: 227-262. Editorial Andrés Bello. Santiago de Chile.

NIEMEYER, H. M. 2013. On the provenience of wood used in the manufacture of snuff trays from San Pedro de Atacama (Northern Chile). J. Archaeol. Sci. 40: 398-404.

NIEMEYER, H. M. \& AGÜERO, C. 2015. Dyes used in pre-Hispanic textiles from the Middle and Late Intermediate periods of San Pedro de Atacama (Northern Chile): new insights into patterns of exchange and mobility. J. Archaeol. Sci. 57: 14-23.

PLANELLA, M. T., C. BELMAR, L. QUIROZ \& D. ESTÉVEZ. 2012a. Propuesta integradora para un estudio del uso de plantas con propiedades psicoactivas en pipas del período Alfarero Temprano y sus implicancias sociales. Revista Chilena de Antropología 5: 91-117.

PLANELLA, M. T., K. COLLAO-ALVARADO, H. M. NIEMEYER \& C. BELMAR. 2012b. Morfometría comparada de semillas de Nicotiana (Solanaceae) e identificación de semillas carbonizadas provenientes de un sitio arqueológico en Chile Central. Darwiniana 50: 207-217.
PLANELLA, M.T., C. BELMAR, L. D. QUIROZ, F. FALABELLA, S. K. ALFARO, J. ECHEVERRÍA \& H M. NIEMEYER. 2015. Towards the reconstruction of the ritual expressions of societies of the Early Ceramic Period in Central Chile: Social and cultural contexts associated with the use of smoking pipes. En: BOLLWERK E. \& S. TUSHINGHAM (eds.), Perspectives on the Archaeology of Pipes, Tobacco and other Smoke Plants in the Ancient Americas. En prensa.

PÉREZ, J. A. \& I. GORDILLO. 1993. Alucinógenos y sociedades indígenas del noroeste argentino. Anales de Antropología 30: 299-350.

POLO, J. M. \& J. DIEZ. 1986. Contribución al atlas palinológico de Andalucía occidental. IV Solanaceae. Lagascalia 14: 45-66.

PUNT, W., P. P. HOEN, S. BLACKMORE, S. A. NILSSON \& A. LE THOMAS. 2007. Glossary of pollen and spore terminology. Rev. Palaeobot. Palynol. 143: 1-81.

QUIROZ, L., C. BELMAR, M. T. PLANELLA, R. MERA \& D. MUNITA. 2012. Estudio de microfósiles de residuos adheridos en pipas cerámicas del sitio JMC-1 Labranza, Región de la Araucanía. Magallania 40: 249-261.

QUIROZ, L. 2014. Colección de referencia de microrrestos de las especies nativas del género Nicotiana en Chile: El caso específico de los tricomas y su morfometría. Taller "Micro Paleoetnobotánica: Relevancia de una Red Interdisciplinaria de Investigaciones en Fitolitos y Almidones", La Paloma, Uruguay, 8 al 12 de diciembre de 2014.

RAFFERTY, S. 2002. Identification of nicotine by gas chromatography/mass spectrometry analysis of smoking pipe residue. J. Archaeol. Sci. 29: 897-907.

RAFFERTY, S. 2006. Evidence of early tobacco in Northeastern North America? J. Archaeol. Sci. 33: 453-458.

SANHUEZA, L., D. BAUDET \& F. FALABELLA. 2004. El Complejo Llolleo mas allá de la vertiente occidental de los Andes. Actas del XVI Congreso Nacional de Arqueología Chilena. Tomo I: 351361. Sociedad Chilena de Arqueología. Museo de Historia Natural de Concepción. Concepción.

STOVEL, E. 2008. Interaction and Social Fields in San Pedro de Atacama, Northern Chile. The Handbook of South American Archaeology 01/2008: 979-1002.

TORRES, C. 1996. Archaeological evidence for the antiquity of psychoactive plant use in the Central Andes. Annali del Museo civico di Rovereto 11: 291-326.

TUSHINGHAM, S., D. ARDURA, J. W. EERKENS, M. PALAZOGLU, S. SHAHBAZ \& O. FIEHN. 2013. Hunter-gatherer tobacco smoking: earliest evidence 
Bol. Soc. Argent. Bot. 51 (1) 2016

from the Pacific northwest coast of North America. J. Archaeol. Sci. 40: 1397-1407.

WILBERT, J. 1987. Tobacco and Shamanism in South America. New Haven, Yale University Press.

WINTER, J. C. 2000. Traditional uses of tobacco by native americans. En: WINTER J. C. (ed.), Tobacco Use by Native North Americans, pp. 9- 58. Norman, University of Oklahoma Press.

WINTER, J.C. 2000a. Food of the Gods: Biochemistry, addiction, and the development of Native American tobacco use. En: WINTER J. C. (ed.), Tobacco Use by Native North Americans, pp. 305-328, Norman, University of Oklahoma Press.

Recibido el 7 de abril de 2015, aceptado el 27 de octubre de 2015 . 